\title{
Dark energy model and its development
}

\section{Commentary}

We know much, but we understand very little, which we feel when we talk about the Universe. But the human mind can go to any length to know the unknown, like the mystery of the universe. Cosmology has made a lot of progress during the last epoch and has at present a substantial observational and experimental basis that confirms that many aspects of the standard cosmological pictures are a good approximation to reality. Still, the empirical basis has not reached the level of precision and accuracy of the Standard Model of particle physics and we cannot talk about a well-established theory of cosmology, in which one can measure the parameters with high precision. With all this, one can say that cosmology is living nowadays a golden epoch and the observational data, which become more and more precise, keeps cosmologists optimistic about establishing a true Standard Cosmological Theory in the near future. If we try to know the origin of the universe then the most accepted model in this direction is the Big Bang theory. The Big Bang theory is an effort to explain what happened at the very beginning of our universe. Discoveries in astronomy and physics have shown beyond a reasonable doubt that our universe did in fact have a beginning. Prior to that moment there was nothing; during and after that moment there was something: our universe. So simply we can say that the big bang theory is an effort to explain what happened during and after that moment. In the early 1990's, one thing was fairly certain about the expansion of the Universe. It might have enough energy density to stop its expansion and collapse. It might have so little energy density that it would never stop expanding, but gravity was certain to slow down the expansion as time went on.

The discovery in 1998 that the universe is not only expanding it is accelerating have caused a sea change in the thinking about the universe. The discovery of cosmic acceleration is arguably one of the most important developments in modern cosmology. But the physical origin of cosmic acceleration remains a deep mystery. According to General Relativity (GR), if the universe is filled with ordinary matter or radiation, the two known constituents of the universe, gravity should lead to a slowing of the expansion. Since the expansion is speeding up, we are faced with two possibilities, either of which would have profound implications for our understanding of the cosmos and of the laws of physics. The first is that $75 \%$ of the universe exists in a new form with large negative pressure, called dark energy. The other possibility is that General Relativity breaks down on cosmological scales and must be replaced with a more complete theory of gravity. Through a tangled history, now the question arises what is the source of dark energy. So it was that cosmological constant could provide a possible source. But this proposal faced a problem that, in the beginning of the universe, it has a very high value. But in the present universe, dark energy density is found to be very low. The question then remains, why has it got the value it has today? To satisfy these extreme values of dark energy, it was proposed that a dynamical model of cosmological constant should be introduced. Rather than dealing directly with the cosmological constant a number of alternative, Cosmologists proposed scalar fields, whose energy density can vary in time and space. Contributions from scalar fields that are constant in space are usually also included in cosmological constant. An
Volume 3 Issue 2 - 2019

\author{
Bharat Borah \\ Department of Mathematics, Dudhnoi College, Dudhnoi, Assam, \\ India
}

Correspondence: Bharat Borah, Department of Mathematics, Dudhnoi College, Dudhnoi, Assam, India,

Email bharatborah@gmail.com

Received: October 30, 2017 | Published: March 14, 2019

incomplete list of scalar field model includes: Quintessence models, $\mathrm{K}$-essence model, tachyon model, and Phantom model and dilatonic models.

There exists another interesting class of dark energy involving a fluid known as a Chaplygin gas, which is termed as Chaplygin gas model. This fluid also leads to the acceleration of the universe at late times. Remarkably, the Chaplygin gas appears like pressure-less dust at early times and like a cosmological constant during very late times, thus leading to an accelerated expansion. Moreover, curvature induced dark energy causing present acceleration is also a very interesting subject in this arena. Despite of many efforts, an interesting attempt, in this direction for probing the nature of dark energy within the framework of quantum gravity is the so called "holographic dark energy" (HDE) proposal. Of all dark energy models, the HDE model takes a unique position because it is an effective theory which introduces an energy density determined by geometric structures of the universe. However, it is found that evolution of the dark energy is same as that of dark matter, and therefore it cannot drive the universe to accelerated expansion. Motivated by this to solve the problem then came Power law Entropy Corrected Holographic Dark energy model and Logarithmic Entropy Corrected holographic dark Energy models by correcting the entropy term of black hole. Along with these, the theoretical variants, Age-graphic Dark Energy model and Ricci Dark Energy model, have also attracted lots of attention. In addition if we omit the issue of growth of structure, we can find a typical example of effective dark energy model is Dvali-Gabadadge-Porrati (DGP) model, which arises from a class of brane world theories in which the gravity leaks out into the bulk at large distance, leading to the accelerated expansion of the universe. But question arises, which one is the most precise one? In present scenario, the most important mission is now to find out which one is the right dark energy model from these so many competing models, which is not an easy task. Alternatively a realistic way is to find out which ones are better in terms of explaining the various astronomical observational data. Such a study with the help of current observational data, can also help us to determine which models can be excluded and to find out with which ones we can continue our farther study.

\section{Acknowledgments}

None.

\section{Conflicts of interest}

The author declares there is no conflict of interest. 